\title{
Lumen
}

Selected Proceedings from the Canadian Society for Eighteenth-Century Studies

\section{Laurent Bordelon face la croyance. Lecture et influence du passé dans le discours contre la superstition (1680-1730)}

\section{Lucie Desjardins}

Volume 29, 2010

URI : https://id.erudit.org/iderudit/1012030ar

DOI : https://doi.org/10.7202/1012030ar

Aller au sommaire du numéro

Éditeur(s)

Canadian Society for Eighteenth-Century Studies / Société canadienne d'étude du dix-huitième siècle

ISSN

1209-3696 (imprimé)

1927-8284 (numérique)

Découvrir la revue

Citer cet article

Desjardins, L. (2010). Laurent Bordelon face la croyance. Lecture et influence du passé dans le discours contre la superstition (1680-1730). Lumen, 29,

117-128. https://doi.org/10.7202/1012030ar

Copyright (c) Canadian Society for Eighteenth-Century Studies / Sociéte canadienne d'étude du dix-huitième siècle, 2010
Ce document est protégé par la loi sur le droit d'auteur. L'utilisation des services d'Érudit (y compris la reproduction) est assujettie à sa politique d'utilisation que vous pouvez consulter en ligne.

https://apropos.erudit.org/fr/usagers/politique-dutilisation/ 


\section{Laurent Bordelon face la croyance. Lecture et influence du passé dans le discours contre la superstition (1680-1730)}

Aux XVII et $\mathrm{XVIII}$ e siècles, on retrouve de nombreux textes qui s'intéressent à la question de la superstition essentiellement pour la combattre, que l'on songe au célèbre Traité des superstitions de Jean-Baptiste Thiers ${ }^{1}$, à l'Histoire critique des pratiques superstitieuses de Pierre Le Brun ${ }^{2}$, à l'Encyclopédie de Diderot et d'Alembert, sans compter les figures de Bayle, de Fontenelle ou encore de Voltaire, dont l'œuvre reste étroitement associée à leur dénonciation. Or, le combat pour la raison est mené sur plusieurs fronts : les grands débats autour de la réalité des faits de sorcellerie et de possession dans les années 1630-1650, ou autour de la nature et de la signification des comètes dans les années 1650-1680, mettent aux prises les savants et $l^{\prime}$ «ignorance» populaire, mais aussi des clercs et des laïcs, selon des stratégies et des solidarités infiniment complexes. Ce sont ces stratégies et ces solidarités que je souhaiterais ici étudier à partir de l'œuvre de Laurent Bordelon, connu surtout pour un roman publié d'abord en 1710, L'histoire des imaginations extravagantes de Monsieur Oufle ${ }^{3}$.

1 Jean-Baptiste Thiers, Traité des superstitions selon l'Écriture sainte, les décrets des conciles et les sentimens des saints Pères et des théologiens, Paris, A. Dezallier, 1679.

2 Pierre Le Brun, Histoire critique des pratiques superstitieuses qui ont séduit les peuples et embarrassé les sçavans, avec la méthode pour discerner les effets naturels d'avec ceux qui ne le sont pas, par un prêtre de l'Oratoire, Paris, J. de Nully, 1702.

3 Laurent Bordelon, L'histoire des imaginations extravagantes de Monsieur Oufle, causée par la lecture de livres qui traitent de la Magie, des Grimoires, des Démoniaques, Sorciers, loups-garous, incubes, succubes et du Sabbat ; des Fées, Ogres, Esprits follets, Gnomes, Fantômes et autres revenants; des Songes, de la pierre philosophales de l'Astrologie judiciaire, des Horoscopes, des talismans, Jours heureux et malheureux, Éclipses, comètes et 
S'il faut en croire le Nouveau dictionnaire historique que Louis Mayeul Chaudon publie en 1789, c'est tout le XVIII ${ }^{\mathrm{e}}$ siècle qui applique à Laurent Bordelon ces quelques vers que Boileau avait adressés quelques décennies plus tôt à Scudéry :

Bienheureux Bordelon, dont la fertile plume

Peut tous les mois sans peine enfanter un volume

Tes écrits, il est vrai, sans art et languissants,

Semblent être formés en dépit du bon sens

Mais ils trouvent pourtant, quoi qu'on en puisse dire,

Un marchand pour les vendre, et des sots pour les lire ${ }^{4}$.

«Son style est si diffus, poursuit Chaudon dans le même esprit, \& si assomant que les compilateurs les plus lourds trouveraient de quoi $\mathrm{s}^{\prime}$ y ennuyer ${ }^{5}$ ", avant de conclure : "Il écrivait pour son plaisir, mais il ne travaillait guère pour celui de ses lecteurs ${ }^{6} »$. Mais l'abbé Chaudon n'est pas le seul dont la critique soit aussi incisive : dans les histoires littéraires, Laurent Bordelon est sans cesse rangé parmi ces précurseurs sans talent dont le début du XVIII ${ }^{e}$ fourmille. Henri Coulet parle ainsi des « romans bizarres » de Bordelon et le Dictionnaire des lettres $d u$ XVII siècle, d'abord paru chez Fayard en 1951, n'a pas jugé bon de revoir l'article consacré à cet auteur au moment de sa mise à jour en 1996. L'entrée, rédigée par un certain Dr. Émile Gérard-Gailly, mentionne que l'auteur a publié "une centaine de volumes hâtifs ou de compilations sur tous les sujets : théologie, astrologie judiciaire, morale, politique, linguistique, biographies, anthologies, traductions, et surtout, récits de voyages extraordinaires, qu'il orna de titres flambants et mystérieux [...]. Si médiocre écrivain qu'il soit, poursuit-il, Bordelon reste un assez

almanachs, enfin de toutes sortes d'apparitions, de sortilèges, d'enchantements, et d'autres superstitieuses pratiques. Le tout enrichi de Figures, et accompagné d'un très grand nombre de notes curieuses, qui rapportent fidèlement les endroits des livres qui ont causé ces imaginations extravagantes, ou qui peuvent servir à les combattre, Paris, N. Gosselin et C. Leclerc, 1710.

4 Boileau, "Satire II", CEuvres complètes, Paris, Gallimard, "Bibliothèque de la Pléiade ", 1979, 77 sq.

5 Louis-Mayeul Chaudon, Nouveau dictionnaire historique; ou, Histoire abrégée de tous les hommes qui se sont fait un nom par des talents, des vertus, des forfaits, des erreurs depuis le commencement du monde jusqu'à nos jours, Caen, G. Leroy, 1789, t. II, p. 185.

6 Ibid.

7 Henri Coulet, Le roman jusqu'à la Révolution, Paris, Armand Colin, 1967, p. 309. 
bon témoin des goûts et des modes littéraires de son temps qu'il flatta et entretint de son mieux ${ }^{8}$ ». Au reste, on doit peut-être à Bordelon luimême les premiers traits de ce portrait peu flatteur, du moins si l'on en juge par certains de ses propos que la critique n'a cessé de reprendre et d'amplifier, alors qu'il se décrit comme un «fort honnête homme \& fort mauvais auteur ${ }^{9}$ ». Déclarant même un jour avec esprit que "ses ouvrages étaient ses péchés mortels, un plaisant lui répliqua que le public en faisait pénitence». $\mathrm{Si}$, à la lumière de ce tableau, il est difficile d'entrevoir les raisons qui poussent Bordelon à écrire autant (il a écrit plus d'une quarantaine de titres), on comprend peut-être mieux cependant pourquoi peu d'études lui ont été consacrées. Signalons pourtant Jacqueline de la Harpe, qui a publié, en 1942, une courte thèse que beaucoup d'articles se plaisent à reprendre ${ }^{10}$; et, plus récemment, Jean-Paul Sermain qui, dans son ouvrage consacré à la réflexivité littéraire ${ }^{11}$, place Bordelon en tête du renouvellement de l'anti-roman, genre littéraire dont les mécanismes et l'importance sont, de nos jours, bien connus.

La valeur attribuée à l'œuvre de Bordelon ne réside pas, à l'évidence, dans la qualité littéraire de l'œuvre, mais plutôt dans sa contribution à l'histoire des idées et des mentalités, ce qu'illustre, en particulier, sa mise en scène de la superstition. En effet, cet oublié de l'histoire littéraire figure généralement en très bonne place dans les compilations et les dictionnaires de sciences occultes qui fleurissent au XIX ${ }^{\mathrm{e}}$ siècle ${ }^{12}$; son œuvre, assure-t-on, constituerait une sorte de catalogue des peurs et des croyances sous l'Ancien Régime. Entre 1707 et 1713, on compte en effet cinq textes dont le but évident est de lutter contre les superstitions : Les oreilles de l'asne d'or ${ }^{13}$, Mital ou Aventures incroyables, et toute-

8 Dictionnaire des Lettres du XVIIe siècle, Fayard [1951], 1996. [Nous soulignons].

9 Il s'agit ici d'un lieu commun, sans cesse repris par la critique depuis le XVIII siècle, mais dont je n'ai pu retracer la source.

10 Jacqueline de la Harpe, L'abbé Bordelon et la lutte contre la superstition en France entre 1680 et 1730, Berkeley et Los Angeles, University of California Press, 1942.

11 Jean-Paul Sermain, Métafictions (1670-1730). La réflexivité dans la littérature d'imagination, Paris, Honoré Champion, 2002.

12 Voir, entre autres, Jacques-Albin-Simon Collin de Plancy, Dictionnaire infernal, ou Bibliothèque universelle, sur les êtres, les personnages, les livres, les faits et les choses qui tiennent aux apparitions, à la magie, au commerce de l'enfer, aux divinations, aux sciences secrètes, Paris, P. Mongie aîné, 1825-1826 [ $2^{\mathrm{e}}$ éd.]

13 Laurent Bordelon, Les oreilles de l'asne d'or, Paris, C. Leclerc, 1707. 
fois, et cætera ${ }^{14}$, Gomgam, ou l'homme prodigieux transporté dans l'air, sur la terre et sous les eaux ${ }^{15}$, L'histoire des imaginations extravagantes de Monsieur Oufle, et Les Tours de Maître Gonin ${ }^{16}$. De ce nombre, L'histoire des imaginations de Monsieur Oufle serait, aux dires Chaudon, le «seul ouvrage de cet abbé qui compte ${ }^{17}{ }^{\prime}$.

Rappelons brièvement la trame. Monsieur Oufle, le héros du livre, est un petit bourgeois, homme marié dont la lecture des livres et notamment des livres de magie, a troublé l'imagination. Dans la suite du roman, Bordelon passe en revue toutes les formes de superstition suivant le même esprit et avec la même ironie. Chaque fois, la superstition semble tirer sa source d'une lecture. Toutefois, si Bordelon fait directement appel à Cervantès dans sa préface, le roman prétend élargir le programme de l'auteur espagnol en englobant, dans sa dénonciation de la fiction, tous les écrits, et non pas uniquement les romans, sa critique s'en prenant même aux ouvrages que l'on considère comme sérieux et qui, contrairement à la fiction, demandent à être lus à la lettre pour les savoirs qu'ils prétendent communiquer.

Le roman est, en effet, étayé par une multitude de sources érudites d'où il tire sa matière. Au tout début du texte, Bordelon nous présente le cabinet de Monsieur Oufle dans lequel se trouvent des tableaux représentant des magiciens, astrologues, devins, sibylles, diables, fantômes et revenants, alors que la galerie attenante, ornée d'un tableau figurant le sabbat, contient toutes sortes d'instruments d'astrologie et d'objets de magie. C'est cette même galerie qui héberge la fameuse bibliothèque de Monsieur Oufle, qui comprend cent dix-huit ouvrages ayant favorisé les dérives superstitieuses du héros. L'auteur nous en dresse la liste complète et accompagne certains titres de commentaires sur l'utilité de ces ouvrages, qui vont de l'Antiquité au tout début du XVIII siècle et appartiennent à différents régimes de discours : histoire, philosophie, cabale, magie, médecine, astronomie, etc. S'y côtoient ainsi les grands titres de la magie et de la démonologie parmi lesquels on retrouve Les Secrets admirables d'Albert le Grand, le Petit Albert, le Malleus Mallefica-

14 Laurent Bordelon, Mital ou Aventures incroyables, et toutefois, et caetera, Paris, C. Leclerc, 1708.

15 Laurent Bordelon, Gomgam, ou l'homme prodigieux transporté dans l'air, sur la terre et sous les eaux, Paris, Veuve G. Saugrain, 1711.

16 Laurent Bordelon, Les tours de Maître Gonin, Paris, C. Leclerc, 1713.

17 Louis-Mayeul Chaudon, Nouveau dictionnaire historique; ou, Histoire abrégée de tous les hommes qui se sont fait un nom par des talents, des vertus, des forfaits, des erreurs depuis le commencement du monde jusqu'à nos jours, op. cit., p. 407. 
rum $^{18}$ dont la première publication remonte à 1486 , le traité célèbre de Martin Del Rio intitulé Les controverses et recherches magiques et publié en 1600, le Tableau de l'inconstance des mauvais anges et des démons de Pierre De Lancre ${ }^{19}$, ouvrage dans lequel, poursuit l'auteur, on trouve une description si étendue du sabbat que «je ne crois pas que l'on y soit mieux introduit que si l'on y avoit été soi-même ${ }^{20}$ ", l'ouvrage de Jean Bodin, La démonomanie des sorciers, publié en 1580; les quelques textes traitant des affaires de possessions démoniaques de la première moitié du XVII siècle (Aix, Loudun, Louviers), trois traités des spectres, l'ouvrage de Gabriel Naudé, $e^{21}$ et ceux plus récents de Bayle ${ }^{22}$ et de Fontenelle ${ }^{23}$, dont Oufle, n'a pas su profiter, sans compter le Traité des superstitions de Jean-Bapstiste Thiers et un roman : Les Entretiens du comte de Gaba$l_{i s}{ }^{24}$ de Montfaucon de Villars. Bref, Monsieur Oufle a une bibliothèque appartenant, en 1708, à un fond bien souvent ancien (peu de ces textes sont republiés après 1679), et ce sera là l'une des causes de ces visions extravagantes. Ce sont en effet tous ces livres, lus sans critique ni jugement, qui ont tourné la tête de Monsieur Oufle. Car, en «vain lisoit-il des ouvrages faits pour combattre ces contes; il ne retenoit seulement dans sa mémoire les histoires qu'il y avoit lues, sans vouloir se laisser persuader par les raisons qui en laissoient connaître la faussetée ${ }^{25}$ ». À cet égard, les anecdotes et les différentes formes de croyances sont nombreuses, trop nombreuses pour être toutes rappelées ici. Aussi voudrais-je aborder la question de la superstition en m'attardant particulièrement à l'une de ses manifestations exemplaires : la croyance

18 Rappelons que Le Malleus Maleficarum («Marteau des sorcières»), de Heinrich Kramer et Jacques Sprenger, publié en 1486, est le traité qu'utilisaient les inquisiteurs pour identifier, confondre et persécuter les sorcières. On compte 14 éditions de ce texte entre 1486 à 1520.

19 Pierre De Lancre, Tableau de l'inconstance des mauvais anges et démons, où il est amplement traicté des sorciers et de la sorcellerie, Paris, J. Berjon, 1612.

20 Laurent Bordelon, Histoire des imaginations extravagantes de Monsieur Oufle, op. cit., p. 12.

21 Gabriel Naudé, Apologie pour tous les hommes qui ont esté faussement soupçonnez de magie, Paris, F. Targa, 1625.

22 Pierre Bayle, Pensées diverses sur les comètes, 1683.

23 Fontenelle, Histoire des oracles, 1687.

24 Nicolas de Montfaucon de Villars, Le comte de Gabalis ou Entretiens sur les sciences secrètes, Paris, C. Barbin, 1670.

25 Laurent Bordelon, Histoire des imaginations extravagantes de M. Oufle, op. cit., p. 14. 
aux revenants et aux spectres. À partir des Imaginations extravagantes de Monsieur Oufle, j'examinerai plus particulièrement le rôle de la tradition et, surtout, les médiations exercées par la culture écrite dans la transmission des savoirs.

Bordelon n'est évidemment pas le premier à aborder la question des revenants, car la croyance en l'existence des êtres surnaturels - du loup-garou au vampire, en passant par le revenant - connait un regain $\mathrm{d}^{\prime}$ intérêt tout au long du XVII ${ }^{\mathrm{e}}$ et dans la première moitié du XVIII ${ }^{\mathrm{e}}$ siècle, comme en témoignent les traités et compilations qui sont publiées au cours de cette période et qui s'intéressent autant aux aspects scientifiques et théologiques du problème qu'à ses dimensions pratiques. Jean Delumeau a, en effet, déjà rappelé de quelle façon la peur est bien souvent liée aux problèmes pratiques que suscite la présence de revenants. Le locataire d'une maison hantée doit-il, par exemple, payer le loyer à son propriétaire? À cette question, Pierre Le Loyer, auteur d'un Discours des spectres ou visions et apparitions d'esprits, comme anges, démons et âmes, se monstrans visibles aux hommes et dont la première édition remonte 1586, répond gravement que

s'il y a une peur juste et légitime des esprits qui hantent une maison, troublent le repos et inquiètent de nuit, que si la peur n'est pas vaine, en ce cas le locataire demeurera quitte des louages demandés, et non autrement si la cause de la crainte ne se trouvait juste et légitime ${ }^{26}$.

Les affaires de revenants sont donc extrêmement délicates. Le Loyer entend même établir une «science des spectres» — c'est son expression - , en dressant une sorte de typologie des différentes apparitions. Mais le Discours des spectres de Le Loyer est loin d'être le seul ouvrage de ce genre : le Traité de l'apparition des esprits ${ }^{27}$ de Noël Taillepied, et les Trois livres des apparitions des esprits, fantosmes, prodiges et accidens merveilleu $x^{28}$ de Loys Lavater; ouvrages savants de la fin du XVI ${ }^{\mathrm{e}}$ siècle qui,

26 Pierre Le Loyer, Discours des spectres ou visions et apparitions d'esprits, comme anges, démons, et âmes, se montrans visibles aux hommes, Paris, N. Buon, 1608, t. II, L. VI, chap. 15. Cité par Jean Delumeau, La peur en Occident, Paris, Fayard, 1978, p. 106.

27 Noël Taillepied, Traité de l'apparition des esprits, à scavoir des âmes séparées, fantosmes, prodiges et accidents merveilleux qui précèdent quelquefois la mort des grands personnages ou signifient changemens de la chose publique, Paris, J. Osmond, 1600.

28 Loys Lavater, Trois livres des apparitions des esprits, fantosmes, prodiges et accidens merveilleux qui souventes fois précèdent la mort de quelque personnage renommé, ou un grand changement ès choses de ce monde composez par Loys Lavater traduits d'Aleman en 
loin d'appartenir à un passé révolu en 1710, se retrouvent, comme par enchantement, dans la bibliothèque de Monsieur Oufle.

La plupart des récits de revenants appartiennent donc à un fonds culturel très ancien qui intègre la mort dans un ensemble plus complexe de croyances et de pratiques dont certaines suscitent la méfiance ou l'hostilité du clergé et des savants. De fait, comme l'a déjà montré Philippe Ariès ${ }^{29}$, $1^{\prime}$ Ancien Régime est parcouru par un ensemble de croyances et de pratiques qui attestent la crainte de voir les morts revenir persécuter les vivants, lorsque les rites nécessaires ne sont pas accomplis. Or, il me semble que l'on assiste pendant tout l'Ancien Régime à une lente transformation de cet univers de récits de revenants, qui se voit de plus en plus pris en charge par une culture écrite qui cherche à les diffuser ou à les dénoncer.

La question des apparitions, des spectres et des fantômes de toutes sortes y occupe une place importante. Avant d'évoquer le cas du personnage de Monsieur Oufle, regardons celui de l'abbé Doudou, le fils aîné du héros, dont le portrait témoigne a contrario de tout l'effort de la philosophie consistant à remettre en cause les jugements faux sur les choses qui procèdent de l'ignorance des phénomènes naturels. L'abbé Doudou est, un «bon garçon qui faisait un mélange très-mal assorti de science et de piété30 $»$. Il était crédule, «au point d'ajouter foi à toutes les histoires qu'on faisoit des sorciers; il n'y avoit pas une apparition quelque étrange qu'elle fût, qui ne lui semblât très possible : aussi étoit-il continuellement dans une grande crainte de voir des fantômes, que rien n'étoit plus affligeant pour lui, rien ne lui donnoit plus d'inquiétude, que d'être obligé de rester seul la nuit dans une chambre ${ }^{31} »$. Ici, ce n'est pas tant la peur qui est la source de la superstition, mais la croyance et l'adhésion aux textes savants qui entraînent une terreur panique chez l'abbé Doudou. Comme le soulignera du reste Bordelon dans un autre texte publié intitulé La Belle éducation, "La crainte et la superstition se soutiennent l'une l'autre ${ }^{32} »$. C'est que les discours qui s'attaquent aux revenants et à la question plus générale de superstition considèrent

françois : plus trois questions proposées et résolues par Pierre Martyr, lesquelles conviennent à cette matière, Genève, [s. é.], 1559.

29 Philippe Ariès, L'homme devant la mort, Paris, Seuil, 1974.

30 Laurent Bordelon, Histoire des imaginations extravagantes de M. Oufle, op. cit., p. 8 [Nous soulignons].

31 Ibid., p. 18 [Nous soulignons].

32 Laurent Bordelon, La belle éducation, [2 éd.], Lyon et Paris, U. Coustellier, p. 170. 
que l'ignorance conduit à la croyance. Ici, les effets de l'ignorance sont sans cesse présentés sous leur aspect le plus ridicule, suivant en cela les Naudé et les Fontenelle et les relectures modernes de la sagesse épicurienne pour laquelle la philosophie a d'abord pour tâche de délivrer l'esprit des terreurs qu'enfantent la peur et l'ignorance. Comme le soulignera plus tard de Jaucourt, qui rédige l'article «Superstition» de l'Encyclopédie, "L'ignorance et la barbarie introduisent la superstition, l'Hypocrisie l'entretient de vaines cérémonies, la faux zèle la répand, \& l'intérêt la perpétue ${ }^{33}{ }^{\prime}$. Suivant le même esprit, le frère de l'abbé Doudou, Sansugue, ne prend position ou ne se prononce pour ou contre les croyances de son père que dans les cas où son intérêt est en jeu. «Quand on lui parloit des diables qui faisaient trouver des trésors, l'eau lui en venoit si fort à la bouche qu'il ne les aurait pas renvoyés, quand même ils lui auroient apparu avec les formes les plus épouventables dont on se sert pour les représenter ${ }^{34}$.» $\mathrm{Si}$ Sansugue ne croit pas aux revenants, ce n'est pas en raison d'une conviction profonde, ni au nom d'une conception moderne de la rationalité, mais tout simplement, nous dit le texte, parce que ceux-ci ne reviennent habituellement sur terre que pour faire des demandes.

Quant au personnage de Monsieur Oufle, il est tout, sauf un ignorant, comme en témoignent les nombreux livres de sa bibliothèque. Aussi la figure de ce docte superstitieux introduit une question qui mérite davantage l'attention. Ouvrons, par exemple, le chapitre intitulé «Discours ou tirade de M. Oufle sur les apparitions», chapitre dans lequel notre héros s'adresse à son frère appelé Noncrède.

Que de Sçavants, dit-il, qui nous apprennent la possibilité de toutes ces apparitions dont vous vous moquez! Que d'historiens qui nous en rapportent des faits incontestables; puisqu'ils sont approuvez, privilégiez \& imprimez! Comment les Phantômes ne seroient-ils pas aussi communs qu'on le dit, puisque les Astres en produisent une infinité. [...] Un des plus illustres philosophes de l'antiquité ne nous assure-t-il pas, que les âmes qui ont vécu dans le dérèglement deviennent des Spectres après leur mort ${ }^{35}$.

Afin de prouver ce qu'il avance, Monsieur Oufle convoque une série de faits divers tirés des ouvrages qu'il possède, de sorte que le roman

34 Laurent Bordelon, Histoire des imaginations extravagantes de M. Oufle, op. cit., p. 9.

35 Ibid., p. 89-90. 
reprend bon nombre d'histoires de revenants répétées à l'envi au fil des âges. Il rapporte, par exemple, qu'en Guinée, on croit que tout vol est perpétré par l'âme des revenants, ou encore l'histoire de l'empereur romain Pertinax qui, deux jours avant d'être tué par les soldats de sa garde, aurait vu dans un étang une figure le menaçant l'épée au poing, ou enfin celle, plus récente et plus célèbre, du marquis de Précy à qui le marquis Rambouillet apparaît pour lui annoncer qu'il vient d'être tué à la guerre.

Bref, la liste des anecdotes où figurent des revenants est longue, si longue que le pauvre Monsieur Oufle l'achève «tout essoufflé», si bien que son frère, Noncrède, en profite pour rétorquer :

Certes, mon frère, vous venez de faire une grande dépense d'érudition. Je n'ai jamais douté que vous n'eussiez beaucoup lu; mais je ne croyois pas que la nature vous eût partagé d'une mémoire aussi fidelle que celle que vous venez de faire paroitre. C'est un grand avantage quand après avoir fait beaucoup de lectures, on s'en ressouvint aussi heureusement que vous. Mais l'avantage seroit bien plus considérable, si le jugement regloit la memoire, c'est-à-dire, si en ressouvenant de tant de choses, on sçavoit en faire \& si on en faisoit en effet un judicieux ouvrage $e^{36}$.

Ce sont tous ces livres, lus sans critique ni jugement, qui ont tourné la tête de Monsieur Oufle. Car en «vain lisoit-il des ouvrages faits pour combattre ces contes; il ne retenoit seulement dans sa mémoire les histoires qu'il y avoit lues, sans vouloir se laisser persuader par les raisons qui en laissoient connaître la fausseté $e^{37}$ "; l'auteur ajoutant, un peu plus loin, «que toute la science, toute la profession, \& même toute la religion du bon homme Oufle étoit renfermée dans les curiosités, dans les tableaux \& dans les livres dont on vient de parler. Et c'est en cela que consistait sont véritable caractère ${ }^{38} »$. Ici, Oufle est en quelque sorte la victime d'une érudition, d'une fausse érudition qui vient s'opposer au bon sens qui, visiblement, n'est pas ici la chose du monde la mieux partagée.

La suite du discours de Noncrède se veut ainsi une critique de ces esprits crédules et une apologie du bon sens. Or, cette crédulité provient d'une faiblesse d'esprit naturelle : c'est du moins l'argument qui 
se déploie dans le texte, argument que l'on trouve généralement dans les discours sur les spectres. Noël Taillepied, dans son Traitté de l'apparition des esprits, affirme que "ceux qui ont la vue mauvaise et croient apercevoir des choses qui ne sont pas réelles. Ceux qui sont faibles ont d'emblée tendance à se persuader qu'ils voient beaucoup de choses épouvantables dont il n'est rien». Il considère néanmoins comme possible et probable l'existence des revenants. Selon lui, les esprits des morts et les revenants se montrent bien souvent aux vivants. Fréquemment écrit-il, les hommes morts sur les champs de bataille réapparaissent dans leur demeure pour faire leurs adieux à leur famille; ils appellent les serviteurs qui les reconnaissent facilement à leur voix. Oufle, rappelons-le, possède ce traité, souscrit à ce qui est écrit, et s'en approprie le contenu, par une activité de reprise et de citation, ne distinguant pas le vraisemblable du possible. Il ne tient donc pas compte de l'argumentation qui se déploie dans les textes qu'il lit, mais en détache simplement quelques anecdotes pour les intégrer à sa perception et à sa croyance. «Je voudrois seulement vous convaincre pour une bonne fois, poursuit Noncrède, \& vous faire reconnaître \& avouer, qu'il n'est point d'un homme d'esprit, d'un homme raisonnable, d'être d'une trop facile crédulité, particulièrement sur cette matière, où l'on a tant de sujets de douter, pour peu que l'on soit instruit de bons principes; $\&$ si bien disposé à distinguer le vray du faux ${ }^{39}$.»

Mais il y a plus. C'est l'autorité même de l'imprimé qui est ici mise en cause. A partir du cas de Monsieur Oufle, on s'aperçoit surtout que la superstition ne relève pas uniquement de ce qu'on pourrait appeler une "culture populaire» susceptible d'être pensée de manière isolée et renvoyée dans les marges du savoir, mais qu'elle suppose une réflexion sur les liens constants qu'elle tisse avec une «culture savante». C'est ce que lui reprochera Noncrède.

Si vous voulez croire absolument tout ce qu'on dit en faveur des Phantômes, des Spectres, des Esprits qui reviennent, des apparitions étranges, dont on fait tant de contes, parce qu'il est imprimé; pourquoi ne croyez vous pas aussi tout ce que l'on a imprimé, pour montrer qu'il ne faut pas ajouter foy à tant d'opinions \& d'histoires, sans connaissance de cause, afin de croire avec raison $\&$ autant que la vérité $l^{\prime}$ exige ${ }^{40}$ 
D'une première manière, le discours de Noncrède insiste sur le rôle et, surtout, l'autorité du livre. Contrairement à Don Quichotte, ce n'est pas tant, comme nous l'avons vu, la lecture d'ouvrages de fiction qui conduit Monsieur Oufle aux dernières extravagances, mais bien celle d'ouvrages savants. L'imprimé vient ici soutenir la croyance, conférant sérieux et crédit aux opinions, et inspirant surtout confiance au lecteur. Mais il faut se méfier de ceux qui parlent au nom du réel, qui en offrent des images de leur invention, flattent les préjugés du lecteur et les confirment par leur autorité, cette tension entre culture de l'imprimé et mystification renvoyant à l'idée suivant laquelle, pour reprendre ici les propos de Jean-Paul Sermain, «L'essai, le traité, et bientôt ces articles que répandent encyclopédie et journaux sont les véhicules modernes de la fable ${ }^{41}$ ». À cette liste, on pourrait bien sûr ajouter la littérature de colportage qui diffuse, sous une forme ou une autre, quelques-uns des ouvrages appartenant à la bibliothèque de $\mathrm{M}$. Oufle que l'on songe par exemple aux adaptations du Grand Albert ou encore aux canards diffusant les cas célèbres de possession et de sorcellerie ${ }^{42}$. Si l'esprit scientifique soumet volontiers ses propres productions à la critique, la civilisation du livre met un public profane à la merci des croyances anciennes dissimulées en savoirs, voire en pseudosciences. Derrière la superstition à l'œuvre dans le récit de Monsieur Oufle se dissimule ainsi toute une réflexion sur l'autorité du livre, et sur la façon dont les auteurs s'érigent automatiquement en savants.

En somme, parallèlement au processus de marginalisation des différentes formes de croyances populaires, on retrouve toujours de nombreuses manifestations savantes qui témoignent de l'importance qu'on leur accorde et qui, sur une large échelle, contribuent à diffuser, pendant tout l'Ancien Régime, des compilations de récits appartenant à la tradition dans lesquels revenants et spectres de tout genre occupent une part importante, que l'on songe au Traité sur les apparitions des esprits et sur les vampires ${ }^{43} \mathrm{~d}^{\prime}$ Augustin Calmet, ou encore au Recueil de dissertations, anciennes et nouvelles, sur les apparitions, les visions et les songes (1751) de Nicolas Lenglet-Dufresnoy. Ces textes, tout comme le roman de Bordelon, montrent bien que les croyances superstitieuses se nourrissent

41 Jean-Paul Sermain, op. cit., p. 282.

42 Voir l'ouvrage de Geneviève Bollème, La Bible bleue, anthologie d'une littérature «populaire», Paris, Flammarion, 1975.

43 Augustin Calmet, Dissertations sur les apparitions des anges, des démons et des esprits, et sur les revenants et vampires de Hongrie, de Bohême, de Moravie et de Silésie, Paris, De Bure l'Ainé, 1746. 
non seulement de la tradition orale ou populaire, mais aussi de représentations provenant de la culture «savante» - que celle-ci soit antique ou moderne - qui recourent à diverses médiations allant des traités aux romans en passant par les histoires tragiques et les pamphlets. $\mathrm{Au}$ modèle popularisé, entre autres, par Malebranche, qui situe l'origine de la superstition dans les dérives de l'imagination commune, s'ajoute ainsi une autre filiation au fur et à mesure que les traités et les compilations accumulent les exemples de la tradition écrite. Dans un contexte où triomphent peu à peu discernement critique, analyse rationnelle et critique des préjugés traditionnels, la question de la superstition suppose dès lors des configurations complexes qui ne sauraient donc se réduire à de simples oppositions entre obscurantisme et lumières.

Cette complexité est d'autant plus manifeste que plusieurs traités tentent bien souvent de ménager un espace entre foi et raison en distinguant un surnaturel «réel» (c'est-à-dire raisonnable) de l'ensemble des superstitions populaires qui sont le fait de l'ignorance. Si la superstition désigne, depuis le Moyen Âge, les excès et dérives de la croyance, que l'Église elle-même cherche à réprimer, elle devient en même temps, à la fin du XVII ${ }^{e}$ siècle et, de façon plus marquée encore, au siècle des Lumières, une catégorie essentielle de l'économie du savoir et de la culture officielle, qui finit par désigner l'altérité face à laquelle la rationalité moderne se définit. L'histoire des imaginations extravagantes de $M$. Oufle participe pleinement de cette rationalité moderne et du processus de marginalisation des croyances dites "populaires» que l'on retrouve à la fin du XVII siècle et pendant la première moitié du XVIII ${ }^{\mathrm{e}}$ siècle. Il y a dès lors lieu de s'interroger sur la fonction d'un discours savant qui dénonce ou, du moins, marginalise la superstition, sans toujours parfaitement abolir les effets de croyance. En effet, les revenants ne sont pas pour autant complètement disparus du discours, comme le montrera avec évidence leur résurgence dans la culture lettrée, notamment gothique ou illuministe, des Lumières finissantes.

\section{LUCIE DESJARDINS}

Université du Québec à Montréal 